\title{
Aprender colocaciones en español: utilización de tres repertorios en estudiantes de español
}

\author{
VIRGINIA DE ALBA QUIÑONES \\ Universidad Pablo de Olavide \\ valbqui@upo.es
}

\section{INTRODUCCIÓN}

El término diccionario es un hiperónimo que engloba distintos tipos de repertorios lexicográficos: glosarios, vocabularios, léxicos, tesoros... Su nacimiento y desarrollo ha estado siempre vinculado al de una obra de consulta - ya sea de un público general o especializado- a la que se recurre para mejorar el uso o la adquisición de una lengua, concretamente del subsistema léxico. Por lo tanto, los diccionarios tienen desde sus orígenes una vocación didáctica que ha condicionado su propia evolución y se erigen como una herramienta indispensable para adquirir no solo la lengua materna (L1 en adelante), sino cualquier lengua extranjera (L2 en adelante).

Pocos ponen en duda lo anteriormente señalado, al menos, respecto de las lexías simples pero, ¿qué sucede con la adquisición de estructuras que trascienden a la palabra y que son una composición constituida por varias? En la Lexicografía española, desde la aparición del Tesoro de la lengua castellana o española (1611), ha sido una tradición la inclusión de algunas de las estructuras plurimembres ${ }^{1}$ que componen las unidades fraseológicas. Sin embargo, hay otras que poco o ningún reflejo han tenido en los diccionarios; una de ellas es el objeto del presente estudio, las colocaciones.

Así, en este trabajo nos planteamos la realización de un estudio empírico que tiene como objetivo indagar si los diccionarios ayudan a los aprendices de español como lengua extranjera ${ }^{2}$ a confeccionar adecuadamente un grupo de colocaciones donde se ha omitido el colocativo. Asimismo, trataremos de discernir si entre los distintos repertorios que se ofertan hay alguno que los informantes identifiquen como el que más útil les resulta o, si por el contrario, todos les parecen igual de provechosos.

\section{ASPECTO TEÓRICO}

Como hemos señalado con anterioridad, en todo el proceso de aprendizaje de una lengua, el diccionario tiene un rol esencial. No obstante, existe una diferencia básica entre el aprendizaje $\mathrm{e}^{3}$ y la adquisición ${ }^{4}$ : la posibilidad de recurrir a un diccionario bilin-

\footnotetext{
${ }^{1}$ Por ejemplo, las paremias y las locuciones.

${ }^{2}$ ELE en adelante.

${ }^{3}$ Como hecho consciente y que se desarrolla desde la voluntad.
} 
güe para el aprendizaje de una lengua extranjera. Este tipo de repertorio lexicográfico usa la L1 del estudiante como vehículo para el aprendizaje de la L2 y suele estar caracterizado porque no aporta mucha información lexicográfica, ya que el objetivo es la consulta rápida: saber lo que significa un término de otra lengua en la L1 y viceversa.

La idea que sirve de base para estos diccionarios es la de ofrecer sinónimos entre lenguas, para lo que se establece una analogía semántica que con mucha frecuencia no puede sustentarse en el uso real de la lengua. No obstante, aunque el anisomorfismo entre lenguas sea un hecho constatable, el diccionario bilingüe es el instrumento más fácil y recurrente para los estudiantes de una lengua extranjera ${ }^{5}$, independientemente de la competencia del aprendiz. No obstante, hay investigadores que inciden en las restricciones del diccionario bilingüe, como Moreno Fernández (1996: 52) cuando señala que «su utilidad es muy limitada para la producción de enunciados [...] la información gramatical, estilística o pragmática es muy limitada» y con el paso del tiempo, el aprendiz no solo necesita comprender mensajes, sino cerrar el entorno conversacional donde nos desarrollamos ${ }^{6}$ y producir mensajes; para esta actividad los diccionarios monolingües de aprendizaje (en adelante DMA) son los más adecuados, según señala Hernández Hernández (2001).

\subsection{Los DMA}

En el transcurso del tiempo, distintas clases de repertorios lexicográficos se han usado para facilitar el aprendizaje de la L2 a los aprendices: nomenclaturas, diálogos, diccionarios bilingües, glosarios... Sin embargo, hasta el siglo XX no surgieron, en el ámbito de la lexicografía anglosajona, los llamados Learner's Dictionaries. En 1899, Sweet centró sus trabajos en los repertorios destinados a aprendices de inglés. No obstante, como señala Hernández Hernández (2001), no fue hasta años más tarde cuando el primero de estos diccionarios apareció, se trató del New Method Dictionary (1935), publicado por la editorial Longman y tras cinco años apareció el General BASIC English Dictionary. Desde entonces, la lexicografía anglosajona ha desarrollado gran cantidad de $\mathrm{DMA}^{7}$. Asimismo, en otras lenguas europeas se elaboraron desde finales del siglo XIX y principios del siglo XX distintos $\mathrm{DMA}^{8}$.

\footnotetext{
${ }^{4}$ Que se produce a nivel subconsciente y que se da en los casos de la/s lengua/s materna/s.

${ }^{5}$ Sus ventajas fueron señaladas por Ruhstaller (2005) y su uso por estudios como el de Corpas Pastor et al. (2001).

${ }^{6}$ Hecho que se concreta en la denominada hipótesis del output de Swain (1985).

${ }^{7}$ Por citar los más importantes en formato papel, señalaremos el Oxford Advanced Learners' Dictionary of Current English (1948), que es una reedición del Idiomatic and Syntactic English Dictionary; el Collins English Language Dictionary (1974); el Logman Dictionary of Contemporary English (1978); el Chambers Universal Leaners' Dictionary (1980); el Harrap's Easy English Dictionary (1980) y, ya más cercanos en el tiempo, el Collins COBUILD English Language Dictionary (1987) o el Cambridge International Dictionary of English (1995).

${ }^{8}$ En la lengua alemana existen varios, como el Großwörterbuch Deutsch als Fremdsprache (1993), el Wörterbuch Deutsch als Fremdsprache (2000) o el PONS Großwörterbuch Deutsch als Fremdsprache (2006) En la lengua francesa, destacamos el Dictionnaire du français langue étrangère (1978) y el Dictionnaire du Français. Référence et apprentissage (1999). Mientras que en la lengua italiana señalamos el Dizionario italiano per stranieri con grammatica della lingua italiana (2001).
} 
Siguiendo a Campos Souto y Pérez Pascual (2003), los DMA son repertorios semasiológicos, sincrónicos, generales (aunque para un grupo de usuarios muy concreto ${ }^{9}$ ), estructurados por orden alfabético directo y monolingües. Dado el público meta al que va dirigido, en los DMA no deberían aparecer arcaísmos ni regionalismos ni tecnicismos ni léxico muy especializado pero, por ejemplo, sí deberían recogerse neologismos o acrónimos de uso frecuente. Actualmente, uno de los criterios más usados en la elección de las entradas es la frecuencia de uso, el léxico más frecuente se recoge tras el análisis de un corpus ${ }^{10}$.

\subsection{Los diccionarios semibilingües o bilinguizados}

Según señala Climent de Benito (2008), cuentan con una larga tradición, no así en la Lexicografía española donde no hemos conseguido encontrar ninguno, aunque sí propuestas, como Ahumada (2006).

Su naturaleza es híbrida, ya que participa de las características tanto del diccionario bilingüe como del DMA. El objetivo de esta obra es sacar de cada una de las que se nutre (el diccionario bilingüe y el DMA) los beneficios, para permitir y facilitar tanto la codificación como la decodificación. De hecho, se concibe como obra de transición y se señala que los diccionarios bilingües serían para aprendices principiantes; los semibilingües, para intermedios; y los DMA, para avanzados. Lo usual es que sea monodireccional $^{11}$, aunque pueden darse otras formas de ofrecer la información, y lo normal es que sigan la secuencia: «entrada en L2 + definición en L2 + correspondencia en la L1».

\section{LOS DMA Y ELE}

Los primeros DMA aparecieron en la última década del siglo pasado. Señalaremos los cuatro que existen en el mercado (por orden cronológico y, en el caso de que hayan sido editados en el mismo año, por orden alfabético):

\subsection{Diccionario para la enseñanza de la lengua española: español para extranjeros}

En 1995 apareció el primer DMA que podemos encontrar en el panorama lexicográfico español, el Diccionario para la enseñanza de la lengua española (Vox en adelante). Se publicó bajo la dirección de Alvar Ezquerra y el equipo de trabajo (formado por docentes del Departamento de Filología de la Universidad de Alcalá de Henares y personas del equipo editorial Biblograf) fue coordinado por Moreno Fernández.

\subsection{Diccionario Salamanca de la lengua española}

Este repertorio fue publicado en el año 1996. Es el resultado del trabajo de un equipo compuesto por personal de la editorial Santillana y docentes de distintas universidades (Salamanca, Barcelona y Tarragona). Los responsables del proyecto son Gutiérrez Cuadrado y Pascual.

\footnotetext{
${ }^{9}$ Aprendices adultos con competencia idiomática solvente en, al menos, una lengua previa.

${ }^{10} \mathrm{Sin}$ embargo, puede resultar que el índice de frecuencia de una voz en el corpus no coincida con el uso o con las necesidades de estos aprendices.

${ }^{11}$ Lo que implica que solo favorece la decodificación.
} 


\subsection{Diccionario de español para extranjeros}

La editorial SM tiene una larga tradición de repertorios lexicográficos con una clara vocación didáctica vinculada al aprendizaje del español como lengua materna. El Diccionario de español para extranjeros (SM en adelante), dirigido por Maldonado (2002), toma como base el diccionario CLAVE (1996), publicado en la misma editorial y también dirigido por la misma lexicógrafa.

\subsection{Diccionario de la lengua española para estudiantes de español}

Este DMA fue editado por Espasa-Calpe y es fruto de un trabajo coordinado por Villar (2002).

\section{LAS COLOCACIONES}

Dado que las unidades que son objeto del presente estudio a lo largo del tiempo han presentado algunos problemas de índole terminológica y han suscitado controversias para su caracterización, hemos seguido a Koike (2001) para resumir las propiedades que presentan estas estructuras. Koike (2001) distingue dos criterios:

- El criterio formal: estas estructuras están formadas por dos o más palabras de frecuente coaparición que está condicionada por el uso y los elementos que la integran pueden ser sustituidos por otros sin que se produzca una variación en su sentido.

- El criterio semántico: la relación entre los elementos que integran las colocaciones no es igualitaria, sino que hay un componente que condiciona la aparición del otro. Por tanto, entre los constituyentes de la colocación se produce un vínculo jerarquizado y entre ambos elementos se establece una relación típica, transparente y cohesionada semánticamente.

Estas características condicionan lo que la Fraseología reconoce como colocaciones. Sin embargo, participamos de la idea de Higueras García (2006), quien señala que en la práctica docente diaria en el aula de ELE se tienen que enseñar otros tipos de unidades que fluctúan entre las combinaciones libres de palabras y las colocaciones, ya que no presentan todas las propiedades que se han señalado con anterioridad para las colocaciones (especialmente de la restricción léxica o la tipicidad). Dado que los informantes de este trabajo son aprendices de ELE, asumimos en la presente investigación la división de Higueras García (2006: 26) de las colocaciones en prototípicas y no prototípicas: «Muchos de los ejemplos que se emplean en actividades no serían consideradas como tales por la mayoría de los lingüistas». Así, pueden encontrarse en la relación de las estructuras colocacionales que integran el corpus de esta investigación unidades que, en la ortodoxia de la Fraseología, no serían asimilables a lo que tradicionalmente se ha venido denominando colocación.

\subsection{Diccionarios de aprendizaje y colocaciones}

Tras el análisis de los cuatro DMA para aprendices de ELE, trabajos anteriores ${ }^{12}$ constataron que las estructuras colocacionales no se recogían de manera sistemática, ya que podían aparecer - si es que lo hacían- en la definición, en el contorno o en los

\footnotetext{
${ }^{12}$ Molina (2010) y de Alba (2014).
} 
ejemplos de los artículos lexicográficos. Asimismo, ambos estudios señalaron a Vox y a $S M$ como los DMA para aprendices de ELE donde más colocaciones aparecían (y este es el motivo de la elección de ambas obras para la realización de esta investigación).

No obstante, el panorama lexicográfico respecto de la combinatoria léxica no es tan desalentador como a primera vista podría parecer: hay obras que se encargan exclusivamente de estas unidades (dejando de lado las disquisiciones terminológicas ${ }^{13}$ ). Algunas ya se han publicado como son los diccionarios REDES: diccionario combinatorio del español contemporáneo (2004) y Diccionario combinatorio práctico del español contemporáneo: las palabras en su contexto (2006, en adelante Práctico) y otras, que hacen uso de las nuevas herramientas que la ingeniería informática nos está ofreciendo, están en desarrollo, como son el proyecto DiCE ${ }^{14}$, el Diccionario de aprendizaje del español como lengua extranjera ${ }^{15}$ o la herramienta ColexWeb (Santana-Suárez et al. 2014).

\section{EL ESTUDIO}

\subsection{Los informantes. Caracterización}

El número de informantes que tomaron voluntariamente parte en esta investigación asciende a $97^{16}$ y son todos alumnos del Centro de idiomas de la Universidad Libre de Berlín ${ }^{17}$. En el mundo globalizado en el que nos desarrollamos, y más en una gran ciudad como Berlín, esto no implica que todos ellos tengan el alemán como L1, o que esta sea su única L1, pero sí que tienen un nivel de competencia lingüística muy alto en esa lengua (tanto como para poder seguir las clases en la universidad). De ellos, 66 eran mujeres y 31 varones; todos ellos mayores de edad, entre los 18 y los 45 años, aunque la inmensa mayoría se sitúa en la horquilla de los 20 y los 25 años.

\subsection{El corpus}

Para la selección de las unidades que forman parte de esta investigación, tomamos como referencia el Plan curricular del Instituto Cervantes. Niveles de referencia para el español (2006, en adelante PCIC), ya que en el apartado de la subcompetencia nocional, aparece un amplio repertorio de lexías simples y distintos tipos de estructuras plurimembres que se hallan dividas según los 6 niveles de dominio lingüístico que determina el Marco Común de Referencia Europeo para las Lenguas (2002, en adelante Marco). Queremos destacar que se han seleccionado exclusivamente aquellas unidades recogidas de manera explícita en los listados que ofrece el PCIC.

El Centro de idiomas de la Universidad Libre de Berlín estructura las clases de L2 siguiendo el enfoque ramificado que ofrece Marco y esto tiene su reflejo en la compo-

\footnotetext{
${ }^{13}$ Bosque (2016).

${ }^{14}$ Disponible en http://www.dicesp.com/paginas

${ }^{15}$ Disponible en http://www.iula.upf.edu/rec/daele/

${ }^{16}$ Fueron seis más los estudiantes que tomaron parte en la realización de la prueba pero que tuvieron que ser eliminados por distintos motivos: origen español o hispano, llegada tarde a la prueba y un problema sensorial en uno de los casos (ceguera).

${ }^{17}$ Quiero manifestar mi agradecimiento más profundo por la participación voluntaria tanto del alumnado como del profesorado del área de español y, especialmente, el incondicional apoyo prestado por la prof. ${ }^{a}$ Graciela Vázquez.
} 
sición del corpus, ya que nos hemos visto obligados, en algunos casos, a combinar estructuras que en los repertorios del PCIC aparecen en un nivel con las del inmediatamente superior ${ }^{18}$. Nos hemos decantado por la estructura colocacional verbo+sustantivo (OD) por ser las unidades de más frecuente uso en el discurso y se ha realizado un extraordinario esfuerzo para tratar de evitar la presencia de estructuras cuyo colocativo fuera o un verbo funcional o el resultado de una traducción entre las lenguas española y alemana. Se recogen a continuación las unidades pluriverbales en relación con los distintos niveles lingüísticos que presentan los informantes:

- B.1.1: cancelar un contrato, celebrar una fiesta, cancelar una visita, servir/poner una copa, anular una cita, dar miedo, poner la mesa, ganar una beca, facturar el equipaje y archivar los documentos.

- B.2.1: montar un negocio, borrar las huellas, convocar una huelga, amueblar un piso, poner una reclamación, romper una relación, hacer un pedido, dar un discurso, quitar la mesa y plantear una duda.

- B.2.2: borrar las huellas, convocar una huelga, poner una reclamación, romper una relación, dar discurso, plantear una duda, donar sangre, confirmar la noticia y saltarse un semáforo.

- C.1.1: experimentar sensaciones, donar sangre, ocultar los sentimientos, dar el pésame, inaugurar el congreso, confirmar la noticia, infundir valor, obtener beneficios y causar un incendio.

- C.1.2: donar sangre, dar el pésame, inaugurar un congreso, confirmar la noticia, saltarse un semáforo, adoptar un comportamiento, mantener la sintonía, obtener una prima, rebatir una hipótesis y asumir la responsabilidad.

\subsubsection{El corpus y su reflejo en los repertorios lexicográficos}

Para la realización del presente trabajo se ha recurrido a dos de los DMA que tienen los aprendices de ELE a su disposición en el mercado (Vox y SM) y a Práctico, un diccionario combinatorio. En cada uno de los niveles, para el diseño de las pruebas se han elegido 10 estructuras plurimembres. Ofrecemos en la tabla que aparece a continuación si estas unidades pueden encontrarse (ya sea en la definición, en el contorno o en los ejemplos) en cada uno de los tres repertorios que hemos utilizado:

\begin{tabular}{|l|l|l|l|}
\hline & VOX & SM & PRÁCTICO \\
\hline B. 1.1 & $2 / 10$ & $4 / 10$ & $8 / 10$ \\
\hline B. 2.1 & $2 / 10$ & $2 / 10$ & $10 / 10$ \\
\hline B. 2.2 & $1 / 10$ & $1 / 10$ & $10 / 10$ \\
\hline C. 1.1 & $4 / 10$ & $2 / 10$ & $10 / 10$ \\
\hline C. 1.2 & $2 / 10$ & $2 / 10$ & $10 / 10$ \\
\hline
\end{tabular}

TABLA 1. Presencia de las colocaciones en los repertorios lexicográficos

\footnotetext{
${ }^{18}$ Por lo tanto y a modo de ejemplo, en el diseño de la investigación para grupo B.1.2 aparecen estructuras que están recogidas en el PCIC los niveles B.1 y B.2.
} 
Los datos no ofrecen lugar a duda alguna, es Práctico el diccionario donde de forma mayoritaria podemos encontrar las unidades que conforman el corpus, concretamente se recogen 48 de las 50 (lo que implica que aparecen el 96\% de las estructuras), mientras que en los otros dos aparecen exclusivamente 11 , lo que representa el $22 \%$ del total posible.

Respecto de las colocaciones, queremos destacar que únicamente hay dos que no se recogen en ninguno de los diccionarios (ambas del nivel B.1.1, estas son cancelar una visita y poner una copa) y que son solo tres las que pueden ser encontradas en todos los repertorios: celebrar una fiesta, tener sensaciones y dar el pésame.

\subsection{El procedimiento}

Para el desarrollo de esta investigación nos ha servido de referente el trabajo realizado por Laufer (2010). Así, se confeccionó un cuestionario (cuestionario 1) donde aparecían 10 enunciados en los que se había elidido el colocativo; la traducción de la colocación a la lengua alemana se encontraba al final entre paréntesis, como podemos ver en los siguientes ejemplos de dos niveles distintos:

B.1.1 El médico está enfermo, ha llamado la enfermera para la cita (einen Termin absagen).

C.1.2 Los resultados del estudio la hipótesis del inicio de la investigación: el experimento ha sido un fracaso (eine Hypothese widerlegen).

En primer lugar, se les dio a los informantes este primer cuestionario y tuvieron 5 minutos para rellenar los huecos que correspondían a los verbos. Transcurrido ese tiempo, se procedió a recopilar el cuestionario 1 y a entregar el dossier con las bases de las colocaciones, los sustantivos, y sus correspondientes artículos lexicográficos en cada uno de los repertorios (pusimos especial cuidado en situar los artículos en distintos lugares siempre y cambiamos aleatoriamente su tamaño para evitar que recurrieran al mismo repertorio por su situación o sus dimensiones). Podemos apreciar cómo quedó la entrada en el siguiente ejemplo con el término beca:

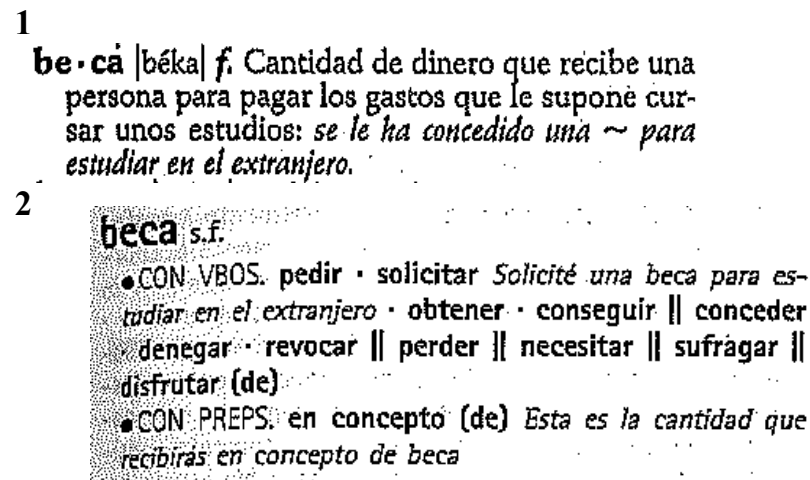

3

be.ca s.f. 1 Ayuda económica temporal que se cor. cede a una persona para que complete sus estrdios o para que realice una investigación o una obra: $H_{t}$ conseguido una beca para investigar los efectos de tabaco. 2 Distintivo honorifico que llevan algunos colegiales como señal de su pertenencia a un deter. minado centro: El último año que estudié en mi co. legio nos impuso las becas el presidente de la fun. dación. 
Asimismo, se les entregó el cuestionario 2, formado exactamente por los 10 enunciados del cuestionario 1 seguidos de distintas cuestiones que reflejamos a continuación:

B.1.1 El médico está enfermo, ha llamado la enfermera para la cita (einen Termin absagen).

¿Has usado el material adicional para rellenar el hueco?

En caso de que sea que sí, en cuál diccionario has encontrado la respuesta:
- Diccionario 1 2 3

— No he encontrado la respuesta en ningún diccionario.

Y se les pidió que volvieran a rellenar los huecos contestando además a las preguntas. Para esta segunda fase dispusieron de 15 minutos, trascurridos los cuales se retiraron ambos documentos.

\section{RESULTADOS Y DISCUSIÓN}

Se reflejan en las tablas que aparecen a continuación los resultados del cuestionario 1 y 2 puestos en relación. Lo primero que queremos destacar es que los datos del primero de los cuestionarios exclusivamente reflejan los fallos cometidos, los aciertos, que son muchos, no son objeto del presente trabajo. Lo segundo que queremos señalar es que hay veces que la columna de las respuestas correctas en el cuestionario 2 no se corresponde con la suma del uso de los diccionarios: esto es así porque en ocasiones los alumnos dicen que sí han usado los diccionarios pero o no los señalan o se han confundido al hacerlo (ya que marcan un repertorio en el que sabemos que no aparece la colocación); igualmente, que hay alumnos - son pocos, concretamente 8 - que señalan que han usado más de un repertorio para saber cuál era el colocativo adecuado (en estos casos, hemos reflejado los dos).

Los datos los hemos estructurado según los niveles de dominio lingüístico que presentaban los grupos de alumnos:

\subsection{El nivel B.1.1}

\begin{tabular}{|l|l|l|l|c|}
\hline \multicolumn{2}{|c|}{ CUESTIONARIO 1 } & \multicolumn{3}{c|}{ CUESTIONARIO 2 } \\
\hline \multirow{2}{*}{ Incorrectas o vacías } & \multirow{2}{*}{155} & Incorrectas/Vacías & \multicolumn{2}{c|}{80} \\
\cline { 3 - 4 } & & & Práctico & 58 \\
\cline { 3 - 4 } & & Correctas (75) & Salamanca & 14 \\
\hline
\end{tabular}

TABLA 2. Relación entre los errores del cuestionario 1 y los resultados del cuestionario 2 en el nivel

Son 24 los alumnos que presentan el nivel más bajo de adquisición de todos los informantes de la muestra. Las colocaciones correctamente confeccionadas tras el uso de los diccionarios suben al $48 \%$ y la inmensa mayoría de los estudiantes señalan que ha usado Práctico para poder elegir bien el colocativo (el 77,3\%) frente a los porcentajes de uso de SM y Vox (el 18\% y el 4\%, respectivamente). 
6.2. El nivel B.2.1

\begin{tabular}{|c|c|c|c|c|}
\hline \multicolumn{2}{|l|}{ CUESTIONARIO 1} & \multicolumn{3}{|l|}{ CUESTIONARIO 2} \\
\hline \multirow{4}{*}{ Incorrectas o vacías } & \multirow{4}{*}{160} & Incorrectas/Vacías & 80 & \\
\hline & & \multirow{3}{*}{ Correctas (80) } & Práctico & 70 \\
\hline & & & SM & 5 \\
\hline & & & Vox & 4 \\
\hline
\end{tabular}

TABLA 3. Relación entre los errores del cuestionario 1 y los resultados del cuestionario 2 en el nivel B.2.1

Los alumnos de este nivel lingüístico son 22. La mejora en el uso de los diccionarios es equivalente a aquellas estructuras que no han sabido construir adecuadamente, ya que de 160 estructuras incorrectas o vacías, justamente la mitad son las que pasan a ser correctas. De nuevo Práctico se erige como el diccionario que los estudiantes señalan como el usado para solventar sus problemas de competencia colocacional, ya que el $87 \%$ de los errores/huecos vacíos han sido corregidos con él, respecto a SM solo el $7,5 \%$ del alumnado marca su uso, mientras que Vox es señalado como el menos utilizado (el 5\%)

\subsection{El nivel B.2.2}

\begin{tabular}{|c|c|c|c|c|}
\hline \multicolumn{2}{|l|}{ CUESTIONARIO 1} & \multicolumn{3}{|l|}{ CUESTIONARIO 2} \\
\hline \multirow{4}{*}{ Incorrectas o vacías } & \multirow{4}{*}{247} & Incorrectas/Vacías & 111 & \\
\hline & & \multirow{3}{*}{ Correctas (136) } & Práctico & 107 \\
\hline & & & SM & 8 \\
\hline & & & Vox & 10 \\
\hline
\end{tabular}

Tabla 4. Relación entre los errores del cuestionario 1 y los resultados del cuestionario 2 en el nivel

Este es el grupo más numeroso de estudiantes, pues lo componen 35 alumnos. Respecto de cómo ha mejorado con el uso de los repertorios bibliográficos, no hay duda alguna: de 247 estructuras incorrectas o vacías, 136 pasan a estar confeccionadas correctamente frente a 111 , lo que equivale al $55 \%$ y al $44 \%$, respectivamente. Si nos centramos en el uso de los diccionarios, nuevamente es Práctico el que los alumnos marcan como el más usado $(83,8 \%)$ frente a SM $(7,3 \%)$ y Vox $(8,8 \%)$

\subsection{El nivel C.1.1}

\begin{tabular}{|c|c|c|c|c|}
\hline \multicolumn{2}{|l|}{ CUESTIONARIO 1} & \multicolumn{3}{|l|}{ CUESTIONARIO 2} \\
\hline \multirow{4}{*}{ Incorrectas o vacías } & \multirow{4}{*}{40} & Incorrectas/Vacías & \multicolumn{2}{|l|}{21} \\
\hline & & \multirow{3}{*}{ Correctas (19) } & Práctico & 14 \\
\hline & & & SM & 3 \\
\hline & & & Vox & 2 \\
\hline
\end{tabular}

TABLA 5. Relación entre los errores del cuestionario 1 y los resultados del cuestionario 2 en el nivel C.1.1

Solo hemos conseguido 12 informantes que presenten un nivel tan alto como para alcanzar un C.1.1. Como en el grupo B.2.1, el uso de las obras lexicográficas condicio- 
na un resultado parejo: las estructuras correctas e incorrectas se distribuyen en partes casi idénticas (19 frente a 21). De nuevo es Práctico el diccionario más usado por los estudiantes (el 73\%); por su parte SM (15\%) y Vox (10,5\%) están muy por debajo del primero en utilización.

6.5. El nivel C.1

\begin{tabular}{|l|l|l|l|l|}
\hline CUESTIONARIO 1 & \multicolumn{2}{l|}{ CUESTIONARIO 2 } \\
\hline \multirow{3}{*}{ Incorrectas o vacías } & \multirow{2}{*}{10} & Incorrectas/Vacías & 4 & \\
\cline { 3 - 4 } & & \multirow{2}{*}{ Correctas (6) } & Práctico & 6 \\
\cline { 3 - 4 } & & SM & 0 \\
\hline & & Vox & 0 \\
\hline
\end{tabular}

TABLA 6. Relación entre los errores del cuestionario 1 y los resultados del cuestionario 2 en el nivel C.1.2

Exclusivamente 4 estudiantes de los que han tomado parte en la investigación alcanzan el nivel C.1.2 $2^{19}$.

Las 10 respuestas incorrectas o vacías en el cuestionario 1, tras el uso del dossier, se transforman en 6 correctas $(60 \%)$ y 4 continúan erradas/vacías. En el uso del diccionario, el $100 \%$ del alumnado señala que ha utilizado Práctico.

Por tanto, dados los datos que arroja el análisis de los cuestionarios, podemos destacar que:

- Todos los grupos han mejorado su competencia colocacional tras el uso de los repertorios que hemos ofrecido. En cifras globales se pasa de 612 elecciones incorrectas/huecos vacíos a 296, lo que supone una reducción por debajo de la mitad de los errores. Queremos destacar que por porcentajes, la horquilla en la que se mueven los distintos grupos oscila entre un subida del índice de aciertos del 60\% (en el grupo C.1.2) y del $48 \%$ (en el nivel C.1.1).

- Los alumnos han señalado mayoritariamente que ha sido Práctico el diccionario que más útil les ha sido para autocorregirse o para saber cuál es el colocativo adecuado, su uso va desde el 73\% del grupo C.1.1 al 100\% del C.1.2. Estos datos ponen de relieve que Práctico es el instrumento que los estudiantes han considerado como el más útil en relación con la competencia colocacional.

\section{CONCLUSIONES}

Los diccionarios han sido desde su concepción un elemento de ayuda para la adquisición de la lengua, ya sea para la L1 o para la L2. En este último caso, encontramos repertorios elaborados para un público meta muy concreto, el de los aprendices de L2, como son: los diccionarios bilingües, los semibilingües y los DMA. Los dos primeros se basan en el establecimiento de paralelismos semánticos entre ambas lenguas que frecuentemente son difíciles de mantener; el tercero es un tipo de repertorio que irrum-

\footnotetext{
${ }^{19}$ Queremos señalar que había más alumnos en este curso, pero que gran parte de ellos eran bilingües (o el español era su L1), por lo que el resultado de sus cuestionarios no ha sido incluido en el presente trabajo.
} 
pió hace poco tiempo en el panorama lexicográfico español, aunque cuenta con una larga tradición en otras lenguas, especialmente, la inglesa.

Para el desarrollo del presente trabajo, nos hemos fijado en una de las unidades que integran el universo fraseológico, las colocaciones. Tradicionalmente estas estructuras no han gozado de gran representatividad en los diccionarios y, en el caso de estar recogidas en ellos, la falta de sistematicidad ha sido una constante. Para el desarrollo de la investigación hemos elegido dos DMA que en estudios anteriores han sido los que más colocaciones recogían. A estos hemos unido Práctico, que es un diccionario combinatorio tal y como se demuestra al comprobar que del corpus de colocaciones que sirve de base para esta investigación el $96 \%$ está registrada en él (frente al $22 \%$ que aparece recogido en los dos DMA).

Tras el desarrollo del estudio empírico, hemos constatado que la competencia colocacional de los estudiantes aumenta con el uso del dossier donde aparecen los diccionarios escaneados, ya que en todo el grupo de informantes se pasa de 612 estructuras erróneamente confeccionadas/vacías en el cuestionario 1, a solo 296 unidades en el cuestionario 2.

Por todo lo anterior, podemos señalar que, según los resultados del estudio llevado a cabo, el uso de los diccionarios ha catalizado un aumento en la elección correcta del colocativo y que, de entre los distintos repertorios, Práctico se erige como el de referencia, ya que los alumnos lo han utilizado mayoritariamente para solventar sus carencias respecto de la adecuada confección de la colocación.

\section{REFERENCIAS BIBLIOGRÁFICAS}

AA. VV. (2001): Dizionario italiano per stranieri con grammatica della lingua italiana, Florencia, Giunti Demetra.

AHUMADA, I. (2006): «El proyecto nebrisense de un diccionario semibilingüe», Hikma: estudios de traducción, 5, pp. 3-13

ALBA QUIÑONES, V. de (2014): «Sobre diccionarios monolingües de aprendizaje del español y colocaciones léxicas. Estudio e implicaciones didácticas», en M. P. Garcés Gómez, ed., Lexicografía teórica y aplicada. Anexos de la Revista de Lexicografia, 26, A Coruña, Universidade da Coruña, pp. 119-134.

Alonso Ramos, M., dir.: Diccionario de Colocaciones del Español. Disponible en http://www.dicesp.com/paginas [consulta: enero de 2016].

AlVAR EzQUeRRA, M., dir. (1995 [2008]): Diccionario para la enseñanza de la lengua española, Barcelona, Universidad Alcalá de Henares y Vox.

BATTANER, P. y S. TORNER, Diccionario de Aprendizaje de Español como Lengua Extranjera. Disponible en http://www.iula.upf.edu/rec/daele/ [consulta: noviembre de 2015].

Best, M. P y J. G. EndicotT (1935): New Method Dictionary, Londres, Longman.

BOSQUE MUÑOZ, I., dir. (2004): Redes. Diccionario combinatorio del español contemporáneo: las palabras en su contexto, Madrid, SM. 
BosQue MUÑOZ, I., dir., (2006): Diccionario combinatorio práctico del español contemporáneo: las palabras en su contexto, Madrid, SM.

Bosque MuÑoz, I. (2015): Entrevista a Ignacio Bosque, Estudios de Lexicografia, 5, pp. 14-42. Disponible en https://issuu.com/ldvp/docs/estudios_de_lexicograf a 5 [consulta: enero 2016].

CAmpos Souto, M. y J. I. PÉRez PAscual (2003): «El diccionario y otros productos lexicográficos» en A. M. Medina Guerra, coord., Lexicografía española, Barcelona, Ariel, pp. 53-78.

CARVer, D., M. WAllance y J. CAMERon (1974): Collins English Language Dictionary, Londres, Harper Collins.

Climent DE BENITO, J. (2008): «Los diccionarios semibilingües: principios y clasificación desde un punto de vista didáctico » en D. Azorín Fernández, dir., El diccionario como puente entre las lenguas y culturas del mundo: Actas del II Congreso Internacional de Lexicografia Hispánica. Disponible en: http://www.cervantesvirtual. com/obra/los-diccionarios-semibilinges-principios-y-clasificacin-desde-un-puntode-vista-didctico-0/ [consulta: enero 2016].

Collin, P. H., ed. (1980): Harrap's Easy English Dictionary, Londres, Harrap.

Corpas Pastor, G., J. Leiva Rojo y M. J. VARela SAlinas (2001): «El papel del diccionario en la formación de traductores e intérpretes: análisis de necesidades y encuestas de uso» en M. Ayala Castro, coord., Diccionarios y enseñanza, Alcalá de Henares, Universidad de Alcalá, pp. 139-173.

Covarrubias Orozco, S. (1611 [2006]): Tesoro de la lengua castellana o española, ed. I. Arellano y R. Zafra, Madrid, Iberoamericana.

DuBois, J. et al. (1978): Dictionnaire du français langue étrangère, París, Larousse.

Götz, D., G. HAENSCH y H. WELLMANN (1993): Großwörterbuch Deutsch als Fremdsprache, Berlín-Münich, Langenscheidt.

Gutiérrez CuAdrado, J., dir. (1996): Diccionario Salamanca de la lengua española, Barcelona, Universidad de Salamanca y Santillana Educación.

HERNÁNDEZ HERNÁNDEZ, H. (2001): «El diccionario en la enseñanza de E.L.E: (Diccionarios de español para extranjeros)», en M. ${ }^{a}$ A. Martín Zorraquino y C. Díez Pelegrín, eds., ¿Qué español enseñar?: norma y variación lingüísticas en la enseñanza del español a extranjeros, Zaragoza, Universidad de Zaragoza, pp. 93-103.

Higueras GARCÍA, M. (2006): Las colocaciones y su enseñanza en la clase de ELE, Madrid, Arco/Libros

InSTITUTO CERVANTES (2006): Plan curricular del Instituto Cervantes. Niveles de referencia para el español. Disponible en http://cvc.cervantes.es/Ensenanza/biblioteca_ele/plan curricular/default.htm [consulta: enero 2016].

KEMPCKE, G. et al. (2000): Wörterbuch Deutsch als Fremdsprache, Berlin-Nueva York, Walter de Gruyter.

KIRKPATRICK, E. M. (1980): Chambers Universal Leaners Dictionary, Edimburgo, Chambers.

KOIKE, K. (2001): Colocaciones léxicas en el español actual, estudio formal y léxico semántico, Alcalá de Henares, Universidad de Alcalá-Takushoku University.

LAUfER, B. (2010): «The Contribution of Dictionary Use to the Production and Retention of Collocations in a Second Language», International Journal of Lexicography, 24, 1, pp. 29-49.

Maldonado, C., dir. (2002): El Diccionario de español para extranjeros, Madrid, SM. 
MINISTERIO DE EDUCACIÓN, CULTURA Y DEPORTE (2002): Marco común europeo de referencia para las lenguas: aprendizaje, enseñanza y evaluación, Madrid, Anaya. Disponible en http://cvc.cervantes.es/ensenanza/biblioteca_ele/marco/ [consulta: abril 2016].

Molina DíAZ, F. (2010): «El tratamiento de las colocaciones en los diccionarios monolingües de español L2», en S. Ruhstaller y M. ${ }^{\mathrm{a}}$ D. Gordón, eds., Diccionario y aprendizaje del español, Berna, Peter Lang, pp. 213-232.

MoRENO FERNÁNDEZ, F. (1996): «El diccionario y la enseñanza del español como lengua extranjera», Cuadernos Cervantes de la lengua española, 2, 11, pp. 47-55.

Nation, I. S. P. (2001): Learning Vocabulary in Another Language, Cambridge, Cambridge University Press.

Ogden, C. K. (1940): The General Basic English Dictionary, Londres, Evans Brothers Limited.

PONS: Deutsch als Fremdsprache Wortebuch. Disponible en http://www.pons.de/ [consulta: febrero 2015].

Procter, P., ed. (1995): Cambridge International Dictionary of English, Cambridge, Cambridge University Press.

Rey-Debove, J., dir. (1999): Dictionnaire du Français. Référence et apprentissage, París, Le Robert.

RUHSTALLER, S. (2005): «Consideraciones sobre los diccionarios monolingüe y bilingüe», en $\mathrm{M}^{\mathrm{a}}$ A. Castillo Carballo et al., coords., La gramática y los diccionarios en la enseñanza del español como segunda lengua: deseo y realidad, Sevilla, Universidad de Sevilla, pp. 86-93.

SANTANA-SUÁREZ, O. et al. (2014): «COLEXWEB, herramienta de consulta de las capacidades combinatorias de las palabras del español», LEA, XXXVI, 2, pp. 273-295. Disponible en: http://www.gedlc.ulpgc.es/art_gied.htm [consulta: mayo de 2015].

SINCLAIR, J. M. et al., eds. (1987): Collins COBUILD English Language Dictionary, Londres, Collins.

Summers, D. y M. RunDELL, eds. (1987): Longman Dictionary of Contemporary English, Londres, Longman.

SWAIN, M. (1985): «Communicative competence: some roles of comprenhensible input and comprenhensible output in its development», en S. M. Gass y C. G. Madden, eds., Input in second language acquisition, Massachussets, Newbury House Publishers, pp. 235-253.

VILLAR, C., coord. (2002): Diccionario de la lengua española para estudiantes de español, Madrid, Espasa.

Título

«Aprender colocaciones en español: utilización de tres repertorios en estudiantes de español» «Learning collocations in Spanish: the use of three lexicons with Spanish students»

Fecha de recepción: 17/06/2016 Fecha de aceptación: 09/12/2016 
Resumen: El estudio trata de analizar la influencia que dos Diccionarios monolingües de aprendizaje y un diccionario combinatorio pueden presentar en el desarrollo de la competencia colocacional léxica en un grupo de 97 aprendices universitarios de español como lengua extranjera con diferentes niveles de domino lingüístico. Para ello, se diseñó una prueba, así se presentaron diez enunciados con colocaciones del tipo verbo + sustantivo (OD) donde se había elidido el colocativo y los estudiantes en dos fases tuvieron que rellenar el hueco: en la primera sin la ayuda de los repertorios lexicográficos y en la segunda haciendo uso de ellos.

Los resultados del presente estudio no dejan lugar a dudas, ya que el dominio colocacional de los estudiantes aumentó de manera considerable en todos los niveles.

Palabras clave: competencia colocacional, colocación léxica, español como lengua extranjera, diccionario.

Abstract: This study investigated the influence that two learners dictionaries and one combinatory dictionary can have in the development of the collocational competence in a group of 97 university students of Spanish as a Foreign Language (with diferent proficiency levels). For that purpose, a lexical task was designed: we give to the students ten sentences with verb-noun collocations and we elaborated a cloze activity (without the verbs). Students had to complete the gaps in two phases: first of all without any support, and afterwards they could use the help of the dictionaries.

Results showed that with the use of the dictionaries, the students' collocational knowledge improved significantly in all levels.

Key words: collocational competence, lexical collocation, Spanish as a Foreing Language, dictionary. 